\title{
Intuition versus analysis: Strategy and experience in complex everyday problem solving
}

\author{
Jean E. Pretz \\ Illinois Wesleyan University, Bloomington, Illinois
}

\begin{abstract}
Research on dual processes in cognition has shown that explicit, analytical thought is more powerful and less vulnerable to heuristics and biases than is implicit, intuitive thought. However, several studies have shown that holistic, intuitive processes can outperform analysis, documenting the disruptive effects of hypothesis testing, think-aloud protocols, and analytical judgments. To examine the effects of intuitive versus analytical strategy and level of experience on problem solving, 1st- through 4th-year undergraduates solved problems dealing with college life. The results of two studies showed that the appropriateness of strategy depends on the problem solver's level of experience. Analysis was found to be an appropriate strategy for more experienced individuals, whereas novices scored best when they took a holistic, intuitive perspective. Similar effects of strategy were found when strategy instruction was manipulated and when participants were compared on the basis of strategy preference. The implications for research on problem solving, expertise, and dual-process models are discussed.
\end{abstract}

When it comes to solving an everyday, practical problem, should the problem be approached analytically or intuitively? When one leads a work team toward achieving a project deadline or makes a decision about where to attend college, will analysis or intuition lead to a better solution? Should one focus on the pros and cons of two alternatives, identify the relevant information, and solve the problem logically, or is it better to rely on an intuitive approach in which one trusts one's feelings and hunches about the situation? Much psychological research on problem solving has attempted to explain how individuals use logic and analysis to solve well-defined problems. However, many everyday, practical problems involve high stakes and are highly complex and ill-structured, lending themselves to a more intuitive approach. Practical problems also involve the application of intuitive, tacit knowledge, which has been gained through experience, rather than through explicit instruction (Sternberg et al., 2000). For practical problems, should we attempt analysis or, instead, rely on intuition? I argue that the answer to this question depends on an individual's level of experience in the domain and his or her preference for thinking intuitively or analytically.

\section{Dual-Process Models}

Recently, psychology has undergone a resurgence of interest in models of cognition highlighting dual processes (e.g., Epstein, 1991; Hogarth, 2001; Sloman, 1996). For example, Epstein's model describes the implicit/intuitive/ experiential mode as holistic, automatic, effortless, affective, slower and more resistant to change, context spe- cific, and passive and preconscious, whereas the explicit/ analytical/rational mode is intentional, effortful, logical, more rapidly and easily changed, context general, and active and conscious. Dual processes have been described with respect to learning (Reber, 1989), memory (Roediger, 1990; Tulving \& Schacter, 1990), and higher cognition, including judgment and decision making (Hogarth, 2001) and reasoning (Sloman, 1996). The present article contrasts dual processes in higher cognition, extending existing research into the domain of problem solving.

Traditionally, the problem-solving literature has focused on how individuals solve well-defined problems, using analytical processes such as means-end analysis and hypothesis testing, which rely on explicit metacognitive processes. Various studies of dual processes in higher cognition have emphasized the benefit of the analytical processing mode in overcoming the heuristic responses of the intuitive-processing mode (e.g., Epstein, Pacini, Denes-Raj, \& Heier, 1996; Greenwald \& Banaji, 1995). Intuitive processing has been often noted as causing errors in judgment, whereas rationality has been held up as an ideal. Intuition has been generally thought of by cognitive psychologists in the decision-making tradition as synonymous with heuristic (e.g., Chase, Hertwig, \& Gigerenzer, 1998; Tversky \& Kahneman, 1974).

However, intuition may not always lead to biased cognition but, instead, may be a powerful tool for thought. Theoretical work on the construct can help to clarify why researchers disagree about the value of intuition. Hill (1987-1988) has described two distinct definitions of in-

J. E.Pretz, jpretz@iwu.edu 
tuition. Classical intuitionism sees intuition as a holisticprocessing mode, whereas inferential intuitionism defines intuition as "a heuristic that represents a logical (inferential) process in which several intermediary steps have been omitted or obscured" (p. 138). This article adopts a classical understanding of intuition. Intuitive thinking is a holistic perspective that takes into account all types of information that often cannot be easily articulated explicitly. Intuition as a holistic process integrates all information without the potentially biasing influence of prior expectations. Holistic intuition is distinct from inferential intuition, which has its roots in an analytical process that has become automatized. Theoretically, analysis and inferential intuition are on a continuum based on automatization, but holistic intuition is thought to be a qualitatively different process altogether.

There is quite a bit of evidence from various literatures in psychology that supports the proposal that classical, holistic intuitive processes outperform analytical processes - for example, in studies of nonverbal communication (Ambady \& Rosenthal, 1992), judgment (Wilson \& Schooler, 1991), decision making (Abernathy \& Hamm, 1995; Dijksterhuis, 2004), and problem solving (Berry \& Broadbent, 1988; Schooler \& Melcher, 1995). Such studies have shown that analytical approaches lead to misleading hypothesis testing or the neglect of relevant information, whereas intuitive approaches allow for a holistic perspective that takes into account all information, regardless of prior hypotheses or schemas.

\section{Dual Processes and Complexity}

Hogarth (2005) has contrasted deliberate (analytical) and tacit (intuitive) processes primarily with regard to judgment and decision-making phenomena. Specifically, he has argued that decisions made on the basis of highly complex inputs will benefit from tacit processing, and those made on the basis of less complex inputs will benefit from deliberate processing. Key to Hogarth's (2005) understanding of the value of intuition is the degree of bias in the environment in which relevant past experience was acquired. If previous experiences involved clear and immediate feedback regarding decision accuracy, the environment is considered kind. Kind environments are conducive to the learning of accurate causal relationships and, thus, facilitate future decision making. In contrast, wicked environments are those in which feedback is unclear and/ or not available in a timely manner. Such learning environments are highly biased, leading the tacit, intuitive system to yield inaccurate decisions in that domain. According to Hogarth (2005), deliberate processing is best when bias is high and complexity is low. Conversely, tacit processing is best when bias is low and complexity is high.

Dijksterhuis and Nordgren (2006) have made similar predictions about the interactive relationship between processing mode and complexity in their theory of unconscious thought. Empirical work has shown that unconscious thought (i.e., thought during a period of distraction or incubation) is superior to conscious thought (i.e., careful analysis of the problem) when the task requires the holistic processing of highly complex stimuli (Dijksterhuis,
2004). This effect was observed most strongly for complex decisions that required the integration of a great deal of information, as opposed to those that required only few pieces of information for a decision to be made. This is due to the weighting principle, the finding that the unconscious is better at calculating weights for numerous factors than is the conscious system, which tends to become biased toward information that is more easily articulated or recently activated.

\section{Dual Processes and Experience}

The fact that processing mode interacts with complexity implies that it will also interact with experience. Complexity and expertise are inversely related; the more experience an individual has, the less complex and more decomposable a problem will appear to that individual. The present work focuses on the role of dual processes and experience in practical problem solving.

Experience affects the organization of knowledge. Expert knowledge is organized according to highly sophisticated schemas, whereas novices lack this deep structure, organizing their knowledge on more surface features (Chi, Feltovich, \& Glaser, 1981). These schemas enable experts to quickly highlight relevant information in a problem and process it quickly and accurately. Schemas facilitate experts' ability to analyze and interpret information in a problem, whereas novices' lack of schemas forces them to rely on other strategies for problem solving (Chi, Glaser, \& Farr, 1988).

In everyday practical problem solving, relevant knowledge is likely to be acquired through informal experience, rather than through direct instruction. Such knowledge is not easily articulated and is referred to as tacit knowledge (Cianciolo, Matthew, Sternberg, \& Wagner, 2006; Polanyi, 1966; Sternberg et al., 2000). Many researchers have described expert knowledge as intuitive in nature (Hogarth, 2001; Klein, 1998). Schön (1983) has explained that experienced leaders form intuitive, implicit, latent representations that reflect patterns of events in the environment acquired through experience. Problems are solved by responding spontaneously and intuitively to a situation by matching it to a similar pattern representation in memory. Experts may engage in this process very successfully, although entirely tacitly.

Sternberg et al. (2000) have developed measures of tacit knowledge that can reveal the presence of expertise even when this knowledge is not easily articulated. Previous research in which these tacit knowledge inventories have been used has shown that individuals with more experience have more tacit knowledge and are more likely to be able to articulate that knowledge. For example, Antonakis, Hedlund, Pretz, and Sternberg (2002) proposed a model of tacit knowledge acquisition and application that highlighted the importance of metacognitive skills in the use of tacit knowledge. The study showed that military officers at higher ranks were distinguished from those at lower ranks by their ability to articulate more detailed metacognitive knowledge in an open-ended measure of tacit knowledge. For example, given a complex scenario, the experts were better able to explicitly identify the main 
problem in the scenario, sift out relevant from irrelevant information, and identify the consequences of possible courses of action. This result suggests that an explicit approach that emphasizes the use of metacognition may enhance practical problem solving among individuals with more experience.

On the basis of the literature reviewed, problem-solving performance should depend on an interaction of strategy and level of experience. Neither an analytical nor an intuitive strategy will be particularly "better" than the other for solving problems. Both Dijksterhuis (2004) and Hogarth (2005) have suggested that processing mode will interact with problem complexity and that complexity and experience can be viewed as inversely related. In fact, Hogarth (2005) has suggested that his proposed interaction of processing mode and complexity should also be examined as an interaction of processing mode and experience. These theories predict that novices will perform best when relying on the tacit system to solve problems. The performance of experienced individuals, on the other hand, will be enhanced to the extent that they use the deliberate system and rely on schemas acquired on the basis of past experience.

\section{The Present Work}

These hypotheses were tested in two studies of college students who solved ill-defined and complex practical problems related to everyday college life. Such problems are often hard to identify and represent clearly, and hardly ever have a clear path to solution. These problems do not lend themselves to decomposition and analysis because the structure of the problem is unclear. It is the process of solving these ill-defined problems in which holistic, implicit cognition is likely to play a role, where an intuitive approach may be more effective than a logical approach (Pretz, 2001).

In order to contrast intuitive and analytical strategies in problem solving, strategy instructions were manipulated. Specifically, participants were taught to rely on either holistic intuition or analysis. Holistic intuition was induced by instructing the participants to bring to mind all relevant information and trust hunches and "tacit" knowledge that might be difficult to explain or rationally justify. In this condition, the participants were also encouraged to incubate by skipping items that were difficult, distracting themselves with other items, similar to Dijksterhuis's (2004) unconscious thought manipulation. Analysis was induced by instructing the participants to define the problem, distinguish relevant from irrelevant information, and monitor problem solving carefully. This metacognitive strategy was based on the metacomponents of the componential subtheory of Sternberg's (1988) triarchic theory of intelligence.

Another way to compare the relative success of either processing mode is to compare individuals who have differences in their preference to engage in one or the other mode. I will refer to individual differences in the preference to think intuitively or analytically as cognitive style. Past research has shown that problem-solving performance depends on an interaction of experience level and cognitive style. For example, Martinsen's (1995) study of insight problem solving showed that participants who preferred to seek novel ways of solving problems (explorers) were most successful with less experience, but that those who preferred traditional problem-solving methods (assimilators) were most successful with more experience. Using both experimental and correlational techniques allows for the discovery of converging evidence in a single design, a research strategy advocated by Cronbach (1957).

Two specific hypotheses are proposed: (1) Problemsolving success depends on an interaction of instructed strategy and experience, and (2) problem-solving success depends on an interaction of cognitive style and experience. Analytical problem solving is expected to be best for participants with sufficient experience in the problemsolving domain to perceive the logic and structure of the problem. Intuitive problem solving is expected to be best for participants with too little experience in the domain to perceive the problems as concrete and well defined. These patterns should be observed whether the strategy is instructed or measured as a cognitive style preference.

\section{STUDY 1}

\section{Method}

\section{Design}

In the present study, an analysis of covariance design was employed. The between-subjects independent variables were manipulated strategy instruction and level of experience. The dependent variable was practical problem-solving performance. Included as covariates in the design were baseline practical-problem-solving ability, cognitive ability, and cognitive style. The participants were assigned to a condition on the basis of the type of strategy instruction they received. One group of participants was taught to use an intuitive approach to problem solving, and a second group an analytical approach. A control group received no instructions regarding strategy use.

\section{Participants}

The participants were 184 Yale undergraduates (118 women, 66 men; mean age $=19.47$ years, $S D=1.10 ; 95$ first-year students and 90 juniors). All of the students participated during the spring semester of the academic year. The students received either credit or cash in return for their participation.

\section{Materials}

Practical problems. The problems were taken from the College Student Tacit Knowledge Inventory (CSTKI; PACE Center, 2002), a 15-item measure of tacit knowledge in the domain of college life. Brief problem scenarios are followed by a series of possible response strategies (usually, approximately eight) that the participant rates in terms of their relative effectiveness on a scale ranging from 1 (extremely bad) to 7 (extremely good). (For a sample item, please see the Appendix.) For more details about the development of these inventories, see Sternberg et al. (2000). In this study, two items from the CSTKI were used as practice items and as illustrations for the strategy instructions. The remaining 13 CSTKI items were used as test items.

Tacit knowledge inventories are scored using a variety of methods that generally compare individual response profiles with an expert or consensus response profile. Researchers have found that nonexpert consensus response profiles and expert response profiles on tacit knowledge inventories are highly correlated (Legree, 1995; Psotka, 2003). Consensus profiles of 1st-year students and juniors in the present study were also highly correlated $(r=.99)$, replicating these previous findings. Problem-solving scores in this study 
were derived by calculating a standardized Euclidean distance (Mahalanobis $D^{2}$ ) of an individual's response profile from the sample consensus response profile. High values on problem solving reflect greater discrepancy from the consensus. The calculation of Mahalanobis distance takes into account the variance and covariance among the ratings in the comparison group. For example, this means that when there is high variability associated with the consensus response for a given response strategy, the distance of an individual rating is weighted less than when variability is low (see Rencher, 1995, for greater detail).

Strategy Use Inventory (SUI). Participants completed the SUI to check actual strategy use in problem solving (see the items in Table 2). Responses on the analytical and intuitive items of the SUI were averaged to obtain a measure of each participant's analytical $(\alpha=.81)$ and intuitive $(\alpha=.58)$ problem-solving strategies.

Analytical and intuitive cognitive styles. To control for individual preferences for strategy use, intuitiveness and analytical thinking as individual-difference, cognitive-style variables were measured using the Rational-Experiential Inventory (REI; Epstein et al., 1996; Pacini \& Epstein, 1999) and the Myers-Briggs Type Indicator (MBTI; Myers, McCaulley, Quenk, \& Hammer, 1998). The REI is a 20-item questionnaire consisting of two 10-item subscales - the rational and experiential inventories. The rational inventory $(\alpha=.78)$ measures the degree to which an individual prefers to rely on logic and analysis in making decisions and solving problems. In contrast, the experiential inventory $(\alpha=.79)$ estimates the degree to which an individual prefers to rely on intuition or hunches when making decisions. The two scales have also been shown to be independent; a person may be high or low on one or both scales.

The MBTI measures individual differences in personality using Jung's (1926) theory of psychological types. The two subscales of the MBTI administered for this study were the intuitive/sensate $(\alpha=$ $.90)$ and the thinking/feeling $(\alpha=.89)$ scales. Intuitive individuals prefer imagination and abstraction, in contrast to sensing individuals, who prefer concrete facts. A thinking type of person is analyti$\mathrm{cal}$, logical, and intellectual, whereas feeling types value emotion and feelings over analysis.

General cognitive ability. Because the practical problem-solving performance used in this study has been found to have weak correlations with measures of $g$ (Cianciolo, Grigorenko, et al., 2006), the participants completed standard measures of fluid intelligence (Cattell Culture Fair Test of $g$, Scale 3, Form A; Cattell \& Cattell, 1961; $\alpha=.64$ ) and crystallized intelligence (Mill Hill Vocabulary Test, Senior Form B; Raven, Raven, \& Court, 1985; $\alpha=.69$ ).

Experience. Level of experience was defined as class year in Yale University. First-year students had had one full semester of college experience, whereas juniors had had at least five semesters of college experience. First-year students were also significantly younger $(M=18.53$ years, $S D=0.54)$ than juniors $(M=20.44$ years, $S D=0.59)[t(174)=22.39, p<.001]$.

\section{Procedure}

Testing sessions lasting $90-120$ min took place in a Yale classroom in groups of 1-20 participants. Each group was randomly assigned to an experimental condition. The procedure was identical for the participants in all of the conditions, except that the control group participants did not receive strategy instructions. The participants first completed the Cattell test at the direction of the experimenter and then completed the Mill Hill test and the cognitive style questionnaires. Then participants completed two practice problemsolving items and filled out the first SUI. The score on these practice items served as the baseline for each student's problem-solving performance. The participants in the experimental conditions were then given the intervention instructions and were asked to complete the test problems, using the instructed strategy. All of the participants completed the SUI after completing half of the problems and again at the end of the problem set. Responses on these two inventories were combined to create a composite SUI score. The participants were then debriefed either as a group or individually.
Analytical strategy instructions. The experimenter gave the participants in the analytical condition explicit instructions on how to use logic and analysis in approaching these practical problems using four steps: (1) Define the problem, (2) identify the relevant pieces of information in the problem, (3) decide how you will use your resources to solve the problem, and (4) identify and evaluate the possible consequences of the potential solutions. After describing these four steps, the experimenter illustrated how the strategy could be implemented in solving each of the two practice problems the participants had just attempted. The participants were instructed to read the scenario and solve the problem using the instructed strategy while rating the quality of the response options given. While solving the test problems, the participants were given a reference handout listing the four steps.

Intuitive strategy instructions. The participants in the intuitive condition were given a set of strategies that encouraged the use of intuition in problem solving. The experimenter defined holistic intuition and encouraged the participants to use the following problemsolving strategies: (1) Imagine the situation vividly, (2) view the problem holistically, (3) trust your hunches and feelings about the problem, and (4) incubate (skip the problem and come back to it later if you get stuck). After presenting these strategies, the experimenter described how they would be used in solving each of the two practice problems. The participants were instructed to read the scenario and solve the problem, using the instructed strategy, while rating the quality of the response options given. The participants also received a handout with the four strategies for their reference.

Control condition. The participants in the control group did not receive any instruction. They were told to solve the problems using whatever strategy felt natural to them.

\section{Results}

Before we conducted the primary analyses, the data were checked for outliers, selection of cognitive style measures, success of the manipulation, and group differences on control variables. The data from 6 participants whose problem-solving scores were at least 3 standard deviations from the mean were removed from the data set. This resulted in a sample with 114 women and 64 men, with 90 first-year students and 88 juniors, and with 62 participants in the analytical condition, 57 in the intuitive condition, and 59 in the control group. The zero-order correlations among cognitive style variables are reported in Table 1. Because the REI experiential and MBTI thinking scales correlated with reported strategy use on the SUI, they were selected as the most valid measures of the intuitive and analytical cognitive styles, respectively.

On the basis of the responses to the SUI (see Table 2), the manipulation check was found to be successful. Overall, the participants reported using the strategy that they were instructed to use. Previous studies in which an experimental manipulation of strategy use has been used have shown that some participants fail to comply with instructions (Sternberg \& Weil, 1980), obscuring the effect of the manipulation. To clarify the effect of the manipulation on problem solving, the participants were classified as either users or nonusers on the basis of their composite score on the SUI for the strategy in which they were instructed. Users were those participants who reported using the instructed strategy above the mean level. There were 97 participants (52 in the analytical condition, 45 in the intuitive condition) who met this criterion for having used the instructed strategy and 23 who did not (10 
Table 1

Study 1: Correlations Among Measures of Cognitive Style and Strategy Use

\begin{tabular}{lcccccc}
\hline \multicolumn{1}{c}{ Subscale } & 1 & 2 & 3 & 4 & 5 & 6 \\
\hline 1. MBTI-thinking & - & $-.22^{* *}$ & .11 & $-.37^{* * *}$ & $.25^{* *}$ & -.12 \\
2. MBTI-intuitive & & - & $.42^{* * *}$ & $.23^{* *}$ & -.10 & .13 \\
3. REI-rational & & & - & .08 & .12 & .07 \\
4. REI-experiential & & & - & -.06 & $.37^{* * *}$ \\
5. Analytical SUI & & & & & - & .08 \\
6. Intuitive SUI & & & & & - \\
\hline
\end{tabular}

Note-MBTI, Myers-Briggs Type Indicator; REI, Rational-Experiential Inventory; SUI, Strategy Use Inventory. ${ }^{* *} p<.01 .{ }^{* * *} p<.001$.

in the analytical condition, 13 in the intuitive condition). All further analyses were conducted on the experimental participants classified as users and on all the participants in the control group.

Univariate ANOVAs were conducted on each covariate as a dependent measure in order to examine any preexisting differences among the participants in the experimental groups. The analyses revealed no main effects or interactions of strategy instruction and level of experience for fluid intelligence, analytical cognitive style, or intuitive cognitive style. There was a main effect of strategy instruction group on crystallized intelligence $[F(2,150)=$ $4.41, p=.01]$. The participants assigned to the intuitive strategy condition scored significantly higher on crystallized intelligence than did the participants in the analytical strategy condition and the participants in the control group. Correlations among the covariates and problemsolving scores are presented in Table A1 of the Appendix. The only covariates that correlated directly with problem solving were fluid intelligence and analytical cognitive style. Higher ability scores were weakly associated with better problem-solving performance, a finding consistent with prior research with these measures (Cianciolo, Grigorenko, et al., 2006). There was a weak relationship between scores on analytical cognitive style and problemsolving success: The participants who preferred analysis tended to do worse on the problem-solving measure.

\section{Major Analyses}

The effects of experience and strategy use (instructed strategy or strategy preference) on problem-solving per- formance were examined using ANCOVAs. The first analysis included experience and instructed strategy condition as independent variables, with problem-solving distance score as a dependent variable. Fluid and crystallized cognitive ability, analytical and intuitive cognitive style, and baseline problem-solving performance were included in the analysis as covariates.

Strategy instructions and level of experience. Hypothesis 1 stated that problem-solving performance depends on an interaction of strategy instruction and level of experience. First-year students were expected to perform better when using an intuitive strategy, and juniors were expected to perform better when using an analytical strategy. The hypothesized interaction of strategy instruction and level of experience was confirmed in the ANCOVA $[F(2,145)=3.63, p=.03]$. The results for the interaction are illustrated in Figure 1. The $y$-axis represents the distance score $\left(D^{2}\right)$ from the consensus on the problem-solving measure. Higher values represent greater discrepancy from the consensus and, thus, less successful performance.

Three covariates (baseline problem-solving score, fluid intelligence, and analytical cognitive style) contributed significantly to the analysis. Zero-order correlations (see Table 1 and the Appendix) showed that baseline scores and fluid intelligence scores were positively related to problemsolving score, and higher analytical style scores were associated with worse problem-solving performance.

The interaction of strategy and experience was due primarily to the effect of the intuitive strategy instructions. First-year students in the intuitive condition $(M=9.85$, $S E=0.67)$ scored significantly better than juniors in the intuitive condition $(M=11.89, S E=0.63)[F(1,145)=$ $5.02, p=.03, d=0.44] .{ }^{1}$ Within the sample of juniors, there was a simple effect of strategy instructions $[F(2,145)=3.42, p=.04]$. Juniors in the intuitive condition $(M=11.89, S E=0.63)$ performed significantly worse than juniors in the analytical condition $(M=9.63$, $S E=0.59)(p=.01, d=0.33)$. The difference between juniors in the intuitive condition and those in the control condition $(M=10.26, S E=0.59)$ was marginal $(p=.06$, $d=0.29$ ). Within the sample of 1st-year students, those using intuition $(M=9.85, S E=0.67)$ scored nonsignificantly better than those in the control group $(M=10.74$, $S E=0.54 ; d=0.24$ ) and those instructed to use analysis

Table 2

Study 1: Postintervention Strategy Use Inventory by Condition

\begin{tabular}{|c|c|c|c|c|c|c|c|}
\hline \multirow[b]{2}{*}{ Strategy } & \multicolumn{2}{|c|}{ Analytical } & \multicolumn{2}{|c|}{ Intuitive } & \multicolumn{2}{|c|}{ Control } & \multirow{2}{*}{$\frac{\text { ANOVA }}{F(2,161)}$} \\
\hline & $M$ & $S D$ & $M$ & $S D$ & $M$ & $S D$ & \\
\hline Imagine the situation very vividly. & 4.37 & 0.72 & 4.56 & 0.66 & 4.23 & 0.87 & 1.36 \\
\hline Break the problem down into steps. & 3.73 & 1.05 & 2.42 & 1.04 & 2.84 & 1.00 & $18.43^{* * *}$ \\
\hline View the problem from a variety of perspectives. & 3.98 & 0.97 & 4.33 & 0.69 & 3.69 & 1.04 & $5.26^{* *}$ \\
\hline Carefully define the problem. & 4.28 & 0.87 & 3.33 & 0.97 & 3.41 & 1.10 & $12.81^{* * * *}$ \\
\hline Rely on guesses or hunches. & 2.60 & 0.85 & 3.88 & 0.97 & 2.85 & 0.95 & $27.98^{* * *}$ \\
\hline Monitor your problem-solving process. & 3.48 & 1.01 & 2.92 & 1.28 & 2.47 & 0.83 & $13.76^{* * *}$ \\
\hline Skip the problem when you are stuck. & 1.71 & 0.93 & 2.49 & 1.25 & 1.86 & 1.12 & $7.20^{* *}$ \\
\hline $\begin{array}{l}\text { Consider information that is implied about the } \\
\text { situation that is not mentioned in the problem } \\
\text { description. }\end{array}$ & 4.09 & 0.83 & 4.41 & 0.73 & 4.11 & 1.00 & 1.17 \\
\hline
\end{tabular}

Note-Strategies rated 1-5 for frequency of use. Intuitive strategies are italicized; analytical strategies are in roman. All participants are included. ${ }^{* *} p<.01 .{ }^{* * *} p<.001$. 
$(M=10.83, S E=0.61 ; d=0.30)$. Overall, the main effects of strategy instruction and level of experience were not significant in this analysis.

The same conceptual hypothesis was also examined among participants in the control group alone. This analysis was used to examine the interaction of experience and strategy preference on the basis of dichotomized cognitive style scores. The prediction was that the more experienced participants scoring above the median on analytical cognitive style would score better than those without a preference for analytical thinking and that less experienced participants scoring above the median on intuitive cognitive style would score better than those who did not report being intuitive.

A $2 \times 2$ ANOVA was conducted for each dichotomized cognitive style variable to examine the effects of cognitive style and complexity on problem-solving scores. Each analysis included baseline problem-solving score and cognitive ability as covariates. Adjusted means for this analysis are reported in Table 3 . The predicted interactions of cognitive style and experience were not found for analytical or intuitive cognitive style, nor was the main effect of experience significant in this set of participants (all $F$ s $<1$ ). Although the expected interaction was nonsignificant, the pattern of means for analytical cognitive style was in line with the hypothesis. Experienced participants with an analytical style tended to perform better than less experienced participants with an analytical style $(d=0.51)$. Experienced students who reported a preference for analytical thinking tended to score better than their low-analytical counterparts $(d=0.44)$. However, contrary to the hypothesis, experienced students who reported a preference for intuition also performed substantially better than those who were less intuitive $(d=0.47)$, with highly intuitive juniors scoring as well as those who were highly analytical.

\section{Discussion}

Overall, the data provided support for Hypothesis 1 and suggested modest support of Hypothesis 2. Hypothesis 1 was confirmed in the significant interaction of experience and strategy instruction among all of the participants. More experienced individuals were more successful when using the instructed analytical strategy than when using the intuitive one. In addition, more experienced participants performed significantly worse when they relied on intuition than when they relied on their own preferred strategy (control group). Furthermore, analysis resulted in worse performance among less experienced, relative to more experienced, participants, and intuition resulted in worse performance among those with more experience, relative to those with less. The expected benefit of intuition for less experienced participants was not found. Performance among inexperienced participants was similar regardless of strategy instructions.

In order to explain this interaction, one must consider that these problems are relatively complex and involve the application of tacit knowledge that is difficult to articulate. Among those with a fair amount of experience,

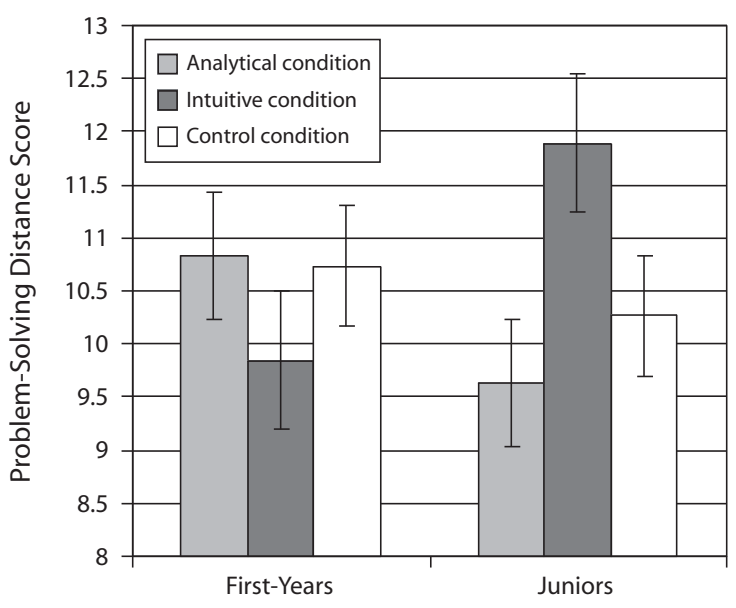

Figure 1. Study 1: Problem-solving performance depends on an interaction of strategy instruction and level of experience. Higher scores reflect worse problem-solving performance. Experimental conditions include users only. Means are adjusted for baseline problem-solving performance, cognitive ability, and cognitive style. Error bars represent standard errors.

analysis aids practical problem solving because it focuses the individual on the information that he or she knows is key to the solution of the problem. When experienced participants rely on intuition, they may be distracted from that which is known to be relevant. Conversely, it is possible that when inexperienced participants attempt analysis, their performance suffers because they are likely to rely on irrelevant information during problem solving. However, the data in this study failed to detect a significant difference between performance of the inexperienced participants in the analytical and intuitive conditions. These explanations are speculative, given that the study provided no evidence to confirm specifically what information was being considered during problem solving. Overall, the data support Hypothesis 1 .

Minimal support was found for Hypothesis 2. There was no interaction of cognitive style and level of experience for either cognitive style. Yet the pattern of means

Table 3

Study 1: Problem-Solving Scores by Level of Experience and Cognitive Style Among Control Group Participants (Adjusted Means)

\begin{tabular}{|c|c|c|c|c|}
\hline \multirow[b]{2}{*}{ Style } & \multicolumn{2}{|c|}{$\begin{array}{c}\text { First-Years } \\
(n=78)\end{array}$} & \multicolumn{2}{|c|}{$\begin{array}{c}\text { Juniors } \\
(n=78)\end{array}$} \\
\hline & $M$ & $S E$ & $M$ & $S E$ \\
\hline High analytical $(n=27)$ & 11.37 & 0.78 & 10.05 & 1.00 \\
\hline Low analytical $(n=32)$ & 10.15 & 0.77 & 10.24 & 0.79 \\
\hline Low intuitive $(n=22)$ & 10.91 & 0.86 & 10.84 & 1.14 \\
\hline High intuitive $(n=37)$ & 10.61 & 0.75 & 9.91 & 0.71 \\
\hline
\end{tabular}

Note-Higher scores reflect worse problem-solving performance. Only control group participants are included. Groups are based on median splits of cognitive style scores. Means are adjusted for baseline problemsolving performance $(M=7.61)$, fluid intelligence $(M=15.36)$, and crystallized intelligence (20.86). 
showed a medium effect of experience on problem solving among participants with an analytical cognitive style. This finding corresponds to the results for Hypothesis 1 . More experienced participants scored better when relying on an analytical strategy or their natural preference for an analytical approach to problem solving. The findings for intuitive cognitive style were inconclusive and did not support the hypothesis. The lack of statistical significance is not surprising, given that this hypothesis tested the effect of an individual-differences independent variable, rather than a manipulated strategy instruction, as tested in Hypothesis 1.

Notably, this study revealed no main effects of strategy instruction, cognitive style, or level of experience. As was expected, neither analysis nor intuition was an optimal strategy for problem solving, whether the strategy was instructed or measured as cognitive style. However, the fact that the participants with more experience were not more successful than those with less experience is problematic. Consequently, in Study 2, additional evidence for the hypotheses was sought, using a sample with a broader range of experience.

One caveat that must accompany the present results regards the content of the problems used. Many of the problems are social and interpersonal in nature, potentially limiting the generalizability of these findings to other domains. However, it is unclear to what extent these results may be influenced by the social nature of the items. In order to address this concern, Study 2 was designed with a more diverse set of problems.

\section{STUDY 2}

The purpose of Study 2 was to replicate the findings of Study 1 with an improved experimental design. In this study, participants on a different campus, with a broader range of expertise, were recruited. Specifically, 1st-year undergraduates were recruited during the first 2 weeks on campus, and this sample was compared with junior and senior undergraduates who had been trained in the supervision of other undergraduates and worked as staff members of the Office of Residential Life. Furthermore, additional problems were developed to sample a broader range of college-related problems, including those that were not social in nature. Finally, validation of the measure was established in two ways. First, items were selected for use on the basis of validity ratings given by supervisors of undergraduate Residential Life staff members. Second, supervisor ratings of performance were sought for all the participants in order to establish a correlation between rated performance and performance on the problem-solving measure. The hypotheses for this study were the same as those in Study 1 . Hypothesis 1 predicted an interaction of strategy instruction and experience, and Hypothesis 2 predicted an interaction of cognitive style and experience.

\section{Method}

\section{Participants}

One hundred nineteen students (43 men, 76 women) from Illinois Wesleyan University participated in the study. First-year students (mean age $=18.04$ years, $S D=0.37$ ) were recruited during their first 2 weeks of their 1st year of college $(n=80)$, and junior and senior students (mean age $=20.77$ years, $S D=0.67$ ), selected for participation on the basis of their experience as a resident assistant (RA) or Greek peer counselor (GPC), were tested throughout the academic year. RAs and GPCs are undergraduates who are responsible for students in residence halls or Greek houses at the university. For simplicity, all RAs and GPCs will be collectively referred to as "Residential Life staff." All staff had received extensive training in dealing with everyday problems that college students face. The students in this sample had received between 120 and $180 \mathrm{~h}$ of training and, on average, had worked for about a year or more in the position (RAs, $M=1.66, S D=0.58, n=29$; GPCs, $M=0.93, S D=0.19$, $n=7)$. Staff interacted frequently with supervisors and received ample feedback regarding their performance, creating an environment conducive to the acquisition of tacit knowledge about problems in college life. All the participants received class credit or a $\$ 10$ gift certificate for their participation.

\section{Materials}

Additional CSTKI problems were developed to better represent a range of problems faced by college students. Eight members of the University's Office of Residential Life, who worked full-time as supervisors of undergraduate staff members, were recruited to evaluate the problems. The supervisors rated the problems to determine which items were most likely to discriminate between students with little experience and those with the kind of experience and training that is characteristic of junior and senior Residential Life staff members. On the basis of this analysis, 10 items were identified that received a rating of 3 (somewhat more experienced than the average student) or above. These items were selected as valid measures of tacit knowledge in college life. Despite efforts to increase the number of items that were less social and interpersonal in nature, the majority of the 10 highly-rated problems dealt with such themes (e.g., living with a thieving roommate, maintaining friendships). Scores were computed by calculating the Mahalanobis distance of each individual's response profile from the consensus of the experienced students in the sample $(\alpha=.82)$. The means of the expert response profile for a sample item are provided in the Appendix.

The same eight supervisors plus 50 staff members were recruited to give ratings of students who participated in the problem-solving study with regard to performance in college life. Staff and supervisors rated the students on a scale of 1 (poor) to 7 (excellent) with regard to decision-making ability; academic skills; interpersonal skills; and ability to balance academics, work, and personal life. Because all the raters were not equally familiar with all of the students in the sample, only those scores from raters who reported a competence level at or above the scale midpoint were included in the analysis. In an effort to establish some convergent validity for the problem-solving measure, problem-solving scores correlated with the composite ratings. Overall, scores on the problem-solving items were correlated significantly with ratings $(r=-.40, p<.05)$. This shows that participants whose problem-solving score was closer to the consensus of the experienced students were also rated as more capable in decision-making and other, related performance factors, confirming that the problems do capture a construct that is related to real-life success in college.

The remaining materials were a subset of those used in Study 1. In the interest of shortening the experimental session, the participants were not tested on cognitive ability, and the REI was the sole measure of analytical and intuitive cognitive style ( $\alpha=.88$ and .90 , respectively).

\section{Procedure}

The procedure for this study was identical to that in Study 1. Groups of participants were randomly assigned to an experimental condition: analysis, intuition, and control. The participants completed the cognitive style measures prior to receiving instruction on problem solving. 


\section{Results}

\section{Preliminary Analyses}

Distance scores on the problem-solving measure were examined for outliers, and all the participants were retained (all scores were within 3 standard deviations of the mean). The effects of strategy and experience on each covariate were then tested in a series of ANOVAs. There were no main effects or interactions with respect to intuitive cognitive style, analytical cognitive style, or baseline problem-solving scores. On the basis of the responses on the SUI, the manipulation was determined to have been successful (see Table 4). The participants were instructed to use a particular strategy. In order to strengthen our confidence in the manipulation, the participants who reported not using the instructed strategy were removed from the primary analyses. This resulted in a sample of 101 participants (66 first-year students and 35 staff). There were 33 participants in the analytical condition, 25 in the intuitive condition, and 43 in the control group.

\section{Major Analyses}

In order to test for the interactive effect of strategy instruction and experience on problem solving, an ANCOVA was conducted. The dependent variable was the mean score on problems that were rated as valid according to the supervisors. Independent variables were strategy instruction condition (analysis, intuition, or control) and level of experience (1st-year students or staff). Covariates included baseline problem-solving score, intuitive cognitive style (REI experiential scores), and analytical cognitive style (REI rational scores). These variables were included because of their correlation with problemsolving scores (see Table A2 of the Appendix for correlation matrix). The adjusted means for the ANCOVA are presented in Figure 2. Higher scores represent worse problem-solving performance.

The results showed a main effect and an interaction, with no significant contribution from the covariates. The main effect of experience $[F(1,92)=4.80, p=.03]$ showed that undergraduate staff members performed better overall $(M=6.65, S D=2.89)$ than did 1st-year students $(M=7.51, S D=2.21)(d=0.33)$. This effect was qualified by an interaction of strategy and experience
$[F(2,92)=3.26, p=.04]$. Simple effects analyses showed that the analytical strategy was significantly more successful for experienced students than for 1st-year students $[F(1,92)=11.12, p=.001, d=1.37]$. Among experienced students, the simple effect of strategy condition was marginal $[F(2,92)=2.55, p=.08]$. The trend showed that among experienced students, analysis led to better performance, as compared with those in the intuition condition $(d=0.82)$ and those in the control group $(d=0.52)$. Intuition led to slightly worse performance, as compared with those in the control group $(d=0.13)$. Among 1 st-year students, there was no simple effect of strategy instruction; the students in all three strategy conditions performed comparably $[F(2,92)=0.95, p=.39]$.

In order to further investigate the reliability of this finding, the participants in the control group were classified as using either an analytical or an intuitive strategy on the basis of their scores on the cognitive style measures. ANCOVAs were conducted on problem-solving scores, using groups created via a median split on REI scores. Cognitive style (above-median vs. below-median scorers) and experience (1st-year students vs. staff) were independent variables, and baseline problem solving was the covariate. Adjusted means are presented in Table 5.

Among control participants, the interaction of analytical cognitive style and experience was found to be significant $[F(1,38)=6.91, p=.01]$. Simple effects analyses showed that staff who were more analytical performed significantly better $(M=6.03, S E=0.68)$ than staff with a low preference for thinking analytically $(M=8.73, S E=1.14)$ $[F(1,38)=4.16, p<.05, d=0.75]$. The difference between highly analytical $(M=8.22, S E=0.65)$ and nonanalytical $(M=6.74, S E=0.57) 1$ st-year students was in the predicted direction, but the significance was marginal $[F(1,38)=2.98, p=.09, d=0.87]$. In a comparison of high-analytical participants at the two levels of experience, staff outperformed 1st-year students $[F(1,38)=5.51, p=$ $.02, d=1.24]$. The same analysis conducted with intuitive cognitive style did not show the expected interaction of cognitive style and experience $[F(1,38)=0.60, p=.44]$. Examining the pattern of means, there was a tendency for all of the participants to score relatively well, with the exception of 1st-year students with low intuitive style. The

Table 4

Study 2: Postintervention Strategy Use Inventory by Condition

\begin{tabular}{|c|c|c|c|c|c|c|c|}
\hline \multirow[b]{2}{*}{ Strategy } & \multicolumn{2}{|c|}{ Analytical } & \multicolumn{2}{|c|}{ Intuitive } & \multicolumn{2}{|c|}{ Control } & \multirow{2}{*}{$\frac{\text { ANOVA }}{F(2,194}$} \\
\hline & $M$ & $S D$ & $M$ & $S D$ & $M$ & $S D$ & \\
\hline Imagine the situation very vividly. & 4.40 & 0.70 & 4.38 & 0.68 & 4.17 & 0.90 & 1.89 \\
\hline Break the problem down into steps. & 3.71 & 0.75 & 2.47 & 1.12 & 2.89 & 1.12 & $24.88^{* * *}$ \\
\hline View the problem from a variety of perspectives. & 4.14 & 0.74 & 4.16 & 0.70 & 3.50 & 1.01 & $13.92^{* * *}$ \\
\hline Carefully define the problem. & 4.12 & 0.66 & 3.27 & 1.10 & 3.49 & 0.96 & $14.76^{* * *}$ \\
\hline Rely on guesses or hunches. & 2.91 & 1.06 & 4.27 & 0.73 & 3.23 & 1.16 & $31.59^{* * *}$ \\
\hline Monitor your problem-solving process. & 3.47 & 0.84 & 2.63 & 1.15 & 2.45 & 0.98 & $20.02^{* * *}$ \\
\hline Skip the problem when you are stuck. & 1.55 & 0.92 & 2.14 & 1.25 & 1.48 & 0.88 & $8.00^{* * *}$ \\
\hline $\begin{array}{l}\text { Consider information that is implied about the } \\
\text { situation that is not mentioned in the problem }\end{array}$ & & & & & & & \\
\hline description. & 3.87 & 0.93 & 4.07 & 0.78 & 3.83 & 0.85 & 1.38 \\
\hline
\end{tabular}

Note-Strategies rated 1-5 for frequency of use. Intuitive strategies are italicized; analytical strategies are in roman. All participants are included. ${ }^{* * *} p<.001$. 


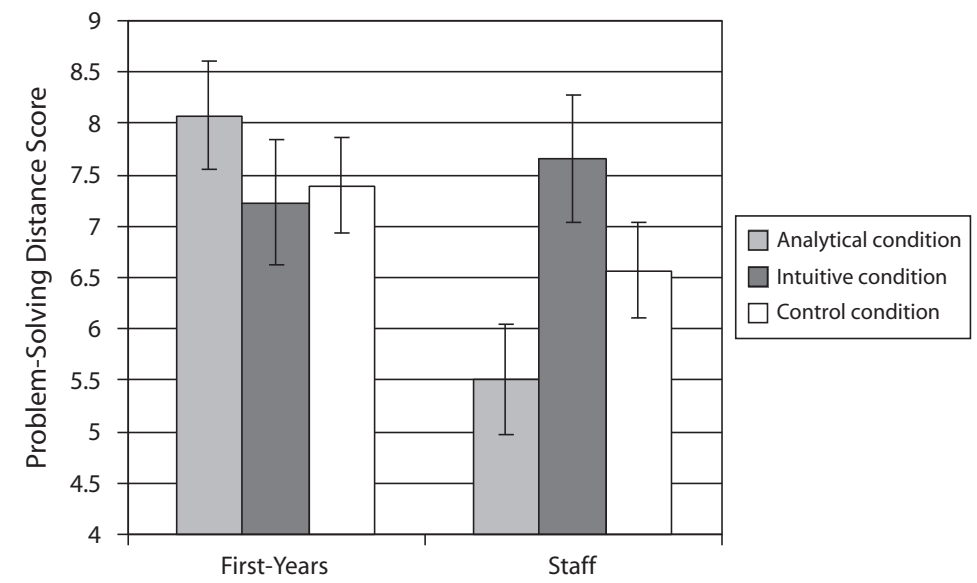

Figure 2. Study 2: Problem-solving performance depends on an interaction of strategy instruction and level of experience. Higher scores reflect worse problem-solving performance. Experimental conditions include users only. Means are adjusted for baseline problem-solving performance and cognitive style. Error bars represent standard errors.

effect of intuitive cognitive style among 1st-year students was large $(d=0.96)$. Notably, staff seemed to do well regardless of their preference for thinking intuitively. In addition, highly intuitive staff scored almost as well as those who were highly analytical. ${ }^{2}$

\section{Discussion}

These results confirm the effects found in Study 1 and provide support for both hypotheses. The interaction of strategy instruction and level of experience was significant, as was found in Study 1, providing further support for Hypothesis 1. Analysis led to the best performance among more experienced participants and the worst performance among those with little experience. The effect of strategy was most pronounced among experienced participants.

In an examination of the performance of the participants in the control group, the pattern of means complemented those found among participants in the experimental conditions, providing support for Hypothesis 2 . The analytical participants with experience performed much better than did the experienced participants who did not prefer

Table 5

Study 2: Problem-Solving Scores by

Level of Experience and Cognitive Style Among Control Group Participants (Adjusted Means)

\begin{tabular}{|c|c|c|c|c|}
\hline \multirow[b]{2}{*}{ Style } & \multicolumn{2}{|c|}{$\begin{array}{l}\text { First-Years } \\
(n=28)\end{array}$} & \multicolumn{2}{|c|}{$\begin{array}{c}\text { Staff } \\
(n=15)\end{array}$} \\
\hline & $M$ & $S E$ & $M$ & $S E$ \\
\hline High analytical $(n=23)$ & 8.22 & 0.65 & 6.03 & 0.68 \\
\hline Low analytical $(n=20)$ & 6.74 & 0.57 & 8.73 & 1.14 \\
\hline Low intuitive $(n=23)$ & 8.29 & 0.68 & 6.82 & 0.72 \\
\hline High intuitive $(n=20)$ & 6.70 & 0.59 & 6.50 & 1.18 \\
\hline
\end{tabular}

Note-Higher scores reflect worse problem-solving performance. Only control group participants are included. Groups are based on median splits of cognitive style scores. Means are adjusted for baseline problemsolving performance $(M=7.72)$. to think analytically. The opposite was true among the less experienced participants; the less analytical thinkers performed better. Although the interaction of intuitive cognitive style and experience did not reach conventional levels of significance, there was a large effect of intuitive preference among less experienced participants. The results of the regression analysis provide converging evidence that higher preference for intuition and lower preference for analysis were associated with better problem solving. Among the staff, there was no evidence for an effect of intuitive cognitive style on problem solving. This suggests that intuition neither helped nor hurt their performance. It was the absence of analysis that led to poor performance among the experienced participants in this study.

The design of Study 2 was significantly improved over that of Study 1. The participants were sampled at a much broader range of experience, including students with 2 weeks of experience and others who had received extensive training in the problem-solving domain. In addition, the problems used were validated by the supervisors of the more experienced participants. Problem-solving scores were determined by distance from a relatively expert consensus, and those scores were moderately correlated with performance ratings.

\section{GENERAL DISCUSSION}

The results of both studies taken together provide substantial evidence for an interaction between level of experience and success of problem-solving strategy. Using analysis improved problem-solving performance among experienced students, and relying on intuition had a small, consistent benefit for inexperienced students. Notably, the findings of both studies showed that neither the intuitive nor the analytical strategy led to better performance than did the other. The appropriateness of the strategy depended on the experience of the problem solver. This interaction can be explained in 
terms of the problem solver's perception of the problem. An experienced individual sees the relevant information in a problem and knows how to analyze it in an explicit, logical manner. Relying on intuition may distract the expert from this critical information. In contrast, an inexperienced individual does not recognize the critical information in the problem and, thus, performs worse when relying on analysis. Instead, an intuitive, holistic approach may facilitate the solution of the problem for the novice by bringing to mind as much relevant information as possible. Additional research could help to confirm these explanations of the effects, perhaps by collecting think-aloud protocols or testing participants' memory for critical pieces of information after the problem-solving task has been completed.

Even when no strategy instructions were given, individual differences in cognitive style had a similar impact on problem solving. Although some of the significance tests for the effects of cognitive style were marginal, this was likely due to the restricted sample size. Effect sizes demonstrated the substantial differences among means. It is difficult to detect effects of an individual-differences variable, especially on a general self-report measure of preferences that may or may not apply to the problems attempted in the study. Another source of inconsistency in these effects may have been the overlap between the median-split groups. Although it was not possible to compare groups that were exclusively high analytical and low intuitive with those that were high intuitive and low analytical due to restricted sample size, the regression analysis for 1st-year students provided some converging evidence in favor of the hypothesis. The fact that any effects were detected lends support to the primary conceptual hypothesis and suggests that the effects may generalize to real-world problem solving in which no specific, pointed strategy is employed.

\section{Implications}

The results of this study may be easily generalized to social problem-solving behavior among undergraduates and, potentially, individuals in other populations, due to the high ecological validity of the materials. The problems used in this study referred to real-life problems encountered by a typical college student, and the instructional interventions were grounded in the context of everyday problem solving. The results of this study have implications for research on problem solving, expertise, and dual processes.

Problem solving. Researchers who aim to enhance practical problem solving and the acquisition of tacit knowledge needed for such tasks must consider that experience level will influence the type of training that will most benefit problem solvers. Research designs that can detect main effects alone may neglect to find meaningful interaction effects such as those found in this study. Other intervention studies seeking to teach problem-solving strategies or enhance decision making should emphasize analytical strategies for individuals with more experience and may find a beneficial effect of a holistic, intuitive approach for novices.

The generalizability of these results may be limited by the content of the practical problems used. As was men- tioned previously, many of the problems used in both studies were social and interpersonal in nature. Notably, evidence from studies of problem solving and judgment that do not involve social elements (Berry \& Broadbent's [1988] studies using a process control task and Wilson \& Schooler's [1991] studies of judgments of strawberry jam) can be interpreted as support for these findings. In fact, social problems may simply be better suited to intuitive problem solving, not because they are social, but because they may be relatively more complex than nonsocial problems. In spite of the effort to broaden the scope of the problem domain in Study 2, the actual problems selected for inclusion in the composite measure of problem solving were relatively social in nature. This effectively eliminated the opportunity to examine the effect of intuition on solving problems that differ in content. In future studies, the problem characteristics should be varied more systematically to further examine the effectiveness of strategies on problems in various domains and with varying levels of complexity.

Expertise. These results imply that experts should use an analytical strategy for solving complex, practical problems. However, this may seem counterintuitive to many experts whose intuitions are highly accurate and based on decades of experience in the domain. In fact, some evidence from the present work showed that when allowed to follow their own preferred strategy, experienced participants who were highly intuitive performed as well as those who were highly analytical. Klein (1998) has shown that expert intuition can be very powerful for complex, high-stakes decision making. Some recent work in the medical decision-making literature suggests that experts' use of analysis may actually disrupt processing, because their knowledge is encapsulated (Wimmers, Schmidt, Verkoeijen, \& van de Wiel, 2005). The results of the work reported here appear to contradict such findings. However, there are two points to be made with respect to this apparent inconsistency. First, the definition of intuition must be clarified. Hill (1987-1988) has made a distinction between holistic and inferential intuition. The novices in this study were instructed to use holistic intuition. In contrast, expert intuition is often considered to be the result of an automatization of previously explicit processes, precisely the kind of intuition that is inferential. Second, it is arguable that the relative experts in Study 2, the more experienced college juniors and seniors, are not actually experts by the definition of the field. As compared with 1st-year students, the juniors and seniors with training may have had a kind of intermediate expertise. In fact, it is difficult to determine who would qualify as an expert in the college life experience on any particular campus. Expertise is said to be achieved generally after 10 years of experience in the domain (Chi et al., 1988), yet this level of experience is rarely achieved by Residential Life fulltime supervising staff members, let alone undergraduate staff members. Future work should attempt to replicate findings in a sample for which clear experts and novices can be identified.

One theoretical model of the development of expertise can reconcile the present findings with evidence that experts 
do well with intuition. Baylor's (2001) U-shaped model of expertise and intuition proposes that intuition is used by both novices and experts but that intermediate experts use a more analytical strategy. As these novices gain knowledge in the domain, they become better able to articulate their knowledge, and as they become intermediate experts who see the structure and logic of problems, they benefit from an analytical strategy. As this knowledge becomes part of an automatized expert schema, they become true experts who can rely on inferential intuition to make decisions and solve problems. Unfortunately, this theory could not be tested in the present study because it did not employ a sufficiently wide range of expertise, as was noted above.

Synthesizing Baylor's (2001) theory with Hogarth's (2005), we would predict a three-way interaction of strategy, experience, and complexity. Baylor's U-shaped relationship between strategy and experience would also depend on complexity, and Hogarth's (2005) predicted interaction of strategy and complexity would also depend on experience. We would predict that even for true experts, extremely complex problems may not be easily analyzed, with intuition resulting in better performance. Similarly, intuition should be available to intermediate experts on relatively simple problems. Future work should test this hypothesized three-way interaction. It must be conducted in a domain in which there exist individuals with very little knowledge and individuals with highly automatized schemas, using problems with varying levels of complexity. This design would test for the predicted interaction and for the distinction between holistic and inferential intuition.

Dual-process models. This theoretical distinction between holistic and inferential intuition has implications for dual-process models. Whereas this study highlights the benefits of intuitive processing, most researchers emphasize the limitations of the implicit, intuitive system. However, these studies criticize solely the heuristic, inferential nature of intuition. The holistic nature of intuition has strong advantages over analytical processing, especially when tasks are so complex that analysis leads to neglect of important information, where the whole is greater than the sum of its parts. Future studies in the dual-process framework should attempt to distinguish between these two types of intuition and their influence on problemsolving and decision-making tasks.

This work contributes evidence to support Hogarth's (2005) current thought on dual processes. I have partially confirmed his predictions with regard to the interaction of processing mode and experience. These results provide evidence that relying on the deliberate system slightly improved performance among experienced individuals, which is in line with Hogarth's (2005) predictions. However, this study does not provide conclusive evidence that inexperienced individuals benefit from tacit processing. Future work should be done to test this theoretical prediction, perhaps with a stronger manipulation of processing mode, such as that used by Dijksterhuis (2004). Additional research is needed to test Hogarth's (2005) prediction about the interaction of strategy and complexity. A recent study has provided evidence for this hypothesis in a different paradigm (Pretz \& Zimmerman, 2007).

\section{Conclusions}

Practical problems are often ill-structured and involve high stakes. This study has demonstrated that the approach to solving such problems depends on a number of factors, including the experience and cognitive style of the problem solver, as well as the complexity of the problem itself. Analysis is a good strategy to the extent that an individual can see the structure of a problem and identify the relevant pieces of information necessary for solution. However, this approach is not beneficial for individuals with little experience.

\section{AUTHOR NOTE}

Study 1 was part of a doctoral dissertation submitted to Yale University. Support for this project was provided by a Yale University Dissertation Fellowship, an APA COGDOP award, and an APA dissertation award. Many thanks to Bob Sternberg, Frank Keil, Geoff Cohen, Jerry Singer, and Jeremy Gray for their helpful comments and suggestions. Study 2 was supported by an Artistic and Scholarly Development grant from Illinois Wesleyan University. Many thanks to Amy Kowalski, Emily Eickhorst, and Sarah Flores for their leadership in data collection, to the Illinois Wesleyan University Office of Residential Life staff for their cooperation in the recruitment process, and to Anna Cianciolo and the reviewers for their invaluable comments on earlier versions of the manuscript. Portions of this work were presented at the 2004 meeting of the Association for Psychological Science and the 2007 meeting of the Cognitive Science Society. Correspondence concerning this article should be addressed to J. E. Pretz, Department of Psychology, Illinois Wesleyan University, P.O. Box 2900, Bloomington, IL 61702-2900 (e-mail: pretz@iwu.edu).

\section{REFERENCES}

Abernathy, C. M., \& Hamm, R. M. (1995). Surgical intuition: What it is and how to get it. Philadelphia: Hanley \& Belfus.

Ambady, N., \& Rosenthal, R. (1992). Thin slices of expressive behavior as predictors of interpersonal consequences: A meta-analysis. Psychological Bulletin, 111, 256-274.

Antonakis, J., Hedlund J., Pretz, J., \& Sternberg, R. J. (2002). Exploring the nature and acquisition of tacit knowledge for military leadership (Research Note 2002-04). Alexandria, VA: Army Research Institute for the Behavioral and Social Sciences.

BAYLOR, A. L. (2001). A U-shaped model for the development of intuition by level of expertise. New Ideas in Psychology, 19, 237-244.

Berry, D. C., \& Broadbent, D. E. (1988). Interactive tasks and the implicit-explicit distinction. British Journal of Psychology, 79, 251-272.

Cattell, R. B., \& Cattell, A. K. S. (1961). Test of " $g$ ": Culture fair. Champaign, IL: Institute for Personality and Ability Testing.

Chase, V. M., Hertwig, R., \& Gigerenzer, G. (1998). Visions of rationality. Trends in Cognitive Sciences, 2, 206-214.

Chi, M. T. H., Feltovich, P. J., \& Glaser, R. (1981). Categorization and representation of physics problems by experts and novices. Cognitive Science, 5, 121-152.

CHI, M. T. H., GlaSer, R., \& FARR, M. J. (1988). The nature of expertise. Hillsdale, NJ: Erlbaum.

Cianciolo, A. T., Grigorenko, E. L., Jarvin, L., Gil, G., Drebot, M. E., \& Sternberg, R. J. (2006). Practical intelligence and tacit knowledge: Advancements in the measurement of developing expertise. Learning \& Individual Differences, 16, 235-253.

Cianciolo, A. T., Matthew, C., Sternberg, R. J., \& Wagner, R. K. (2006). Tacit knowledge, practical intelligence, and expertise. In K. A. Ericsson, N. Charness, P. J. Feltovich, \& R. R. Hoffman (Eds.), The Cambridge handbook of expertise and expert performance (pp. 613-632). New York: Cambridge University Press.

CoHEN, J. (1988). Statistical power analysis for the behavioral sciences (2nd ed.). Mahwah, NJ: Erlbaum.

Cronbach, L. J. (1957). The two disciplines of scientific psychology. American Psychologist, 12, 671-684.

Disksterhuis, A. (2004). Think different: The merits of unconscious thought in preference development and decision making. Journal of Personality \& Social Psychology, 87, 586-598. 
Disksterhuis, A., \& Nordgren, L. F. (2006). A theory of unconscious thought. Perspectives on Psychological Science, 1, 95-109.

Epstein, S. (1991). Cognitive-experiential self-theory: An integrative theory of personality. In R. C. Curtis (Ed.), The relational self: Theoretical convergences in psychoanalysis and social psychology (pp. 111-137). New York: Guilford.

Epstein, S., Pacini, R., Denes-RaJ, V., \& Heier, H. (1996). Individual differences in intuitive-experiential and analytical-rational thinking styles. Journal of Personality \& Social Psychology, 71, 390-405.

Greenwald, A. G., \& BanaJI, M. R. (1995). Implicit social cognition: Attitudes, self-esteem, and stereotypes. Psychological Review, 102, 4-27.

HiLL, O. W. (1987-1988). Intuition: Inferential heuristic or epistemic mode? Imagination, Cognition, \& Personality, 7, 137-154.

Hogarth, R. M. (2001). Educating intuition. Chicago: University of Chicago Press.

Hogarth, R. M. (2005). Deciding analytically or trusting your intuition? The advantages and disadvantages of analytic and intuitive thought. In T. Betsch \& S. Haberstroh (Eds.), Routines of decision making (pp. 67-82). Mahwah, NJ: Erlbaum.

Jung, C. J. (1926). Psychological types. London: Routledge \& Kegan Paul.

KLEIN, G. (1998). Sources of power: How people make decisions. Cambridge, MA: MIT Press.

LEgreE, P. J. (1995). Evidence for an oblique social intelligence factor established with a Likert-based testing procedure. Intelligence, 21, 247-266.

Martinsen, O. (1995). Cognitive styles and experience in solving insight problems: Replication and extension. Creativity Research Journal, 8, 291-298.

Myers, I., McCaulley, M. H., QuenK, N. L., \& Hammer, A. L. (1998). Manual: A guide to the development and use of the Myers-Briggs Type Indicator (2nd ed.). Palo Alto, CA: Consulting Psychologists Press.

PACE Center (2002). College Student Tacit Knowledge Inventory [Unpublished test].

PACINI, R., \& Epstein, S. (1999). The relation of rational and experiential information processing styles to personality, basic beliefs, and the ratio-bias phenomenon. Journal of Personality \& Social Psychology, 76, 972-987.

Polanyi, M. (1966). The tacit dimension. Garden City, NY: Doubleday.

PretZ, J. E. (2001). Implicit processing aids problem solving: When to incubate ideas and trust intuitions. Unpublished manuscript.

Pretz, J. E., \& Zimmerman, C. (2007, August). Rule discovery in the balance task depends on strategy and rule complexity. Poster presented at the 29th Annual Conference of the Cognitive Science Society. Nashville, TN.

PsotKa, J. (2003, December). Consensual scaling of informal knowledge. Talk presented at the Abilities and Expertise Seminar Series in the Yale Psychology Department, New Haven, CT.

Raven, J. C., Raven, J., \& CourT, J. H. (1985). Mill Hill Vocabulary Scale. San Antonio, TX: Harcourt Assessment.
ReBER, A. S. (1989). Implicit learning and tacit knowledge. Journal of Experimental Psychology: General, 118, 219-235.

RENCHER, A. C. (1995). Methods of multivariate analysis. New York: Wiley.

ROEDIGER, H. L., III (1990). Implicit memory: Retention without remembering. American Psychologist, 45, 1043-1056.

ScHöN, D. A. (1983). The reflective practitioner: How professionals think in action. New York: Basic Books.

Schooler, J. W., \& Melcher, J. (1995). The ineffability of insight. In S. M. Smith (Ed.), Creative cognition approach (pp. 97-133). Cambridge, MA: MIT Press.

Sloman, S. A. (1996). The empirical case for two systems of reasoning Psychological Bulletin, 119, 3-22.

Sternierg, R. J. (1988). The triarchic mind. New York: Penguin.

Sternberg, R. J., Forsythe, G. B., Hedlund, J., Horvath, J., SNOOK, S., Williams, W. M., ET AL. (2000). Practical intelligence in everyday life. New York: Cambridge University Press.

SternberG, R. J., \& Weil, E. M. (1980). An aptitude * strategy interaction in linear syllogistic reasoning. Journal of Educational Psychology, 72, 226-239.

Tulving, E., \& Schacter, D. L. (1990). Priming and human memory systems. Science, 247, 301-306.

TVERSKy, A., \& KaHNEMAN, D. (1974). Judgment under uncertainty: Heuristics and biases. Science, 185, 1124-1131.

Wilson, T. D., \& Schooler, J. W. (1991). Thinking too much: Introspection can reduce the quality of preferences and decisions. Journal of Personality \& Social Psychology, 60, 181-192.

Wimmers, P. F., Schmidt, H. G., Verkoeijen, P. P. J. L., \& Van de WIEL, M. W. J. (2005). Inducing expertise effects in clinical case recall. Medical Education, 39, 949-957.

\section{NOTES}

1. Cohen's $d$ (Cohen, 1988) is reported for critical mean comparisons based on the unadjusted means. This method of calculation was chosen because no corresponding standard deviation was available for the adjusted means.

2. In order to better understand the relative contribution of both cognitive style variables simultaneously, a hierarchical regression analysis was conducted for participants at each level of experience. Baseline performance was entered in the first block, and both cognitive style variables were entered simultaneously into the second block. Among 1st-year students, both intuitive ( $\beta=-.38, p=.03)$ and analytical $(\beta=$ $.36, p=.03)$ cognitive styles were significant independent predictors of problem-solving performance. The best problem-solving scores were associated with higher intuitive cognitive style and lower analytical cognitive style for the 1st-year sample. Together, the two cognitive style variables explained $31.8 \%$ of the variance in problem-solving scores above and beyond that contributed by baseline scores. The same analysis was conducted for staff, but the results were not significant.

\section{APPENDIX \\ Sample Problems}

\section{Sample Problem Including Mean Ratings for Each Response Strategy Across All Participants in Study 1}

You have decided to apply for an internship during the upcoming break, and want to ask one of your professors for a letter of recommendation. The professor you have in mind is teaching a fairly large class, and he does not know you very well. One day you run into him in the coffee shop, where he is sitting with what you assume are his kids. Given this situation, rate the quality of the following options:

\begin{tabular}{|ccccccc|}
\hline 1 & 2 & 3 & 4 & 5 & 6 & 7 \\
---+--- & ----+--- & ---+---- & ---+--- & ----+--- & ---++--- & ----+--- \\
Extremely & Very & Somewhat & Neither Bad & Somewhat & Very & Extremely \\
Bad & Bad & Bad & Nor Good & Good & Good & Good \\
\hline
\end{tabular}

3.20 a) You decide that this is a good time to talk to him about the letter.

5.51 b) You go up and greet him, reminding him of your name and what class you are in.

4.13 c) You greet him and then start chatting with his kids.

3.69 d) You nod but do not talk to him. 


\section{APPENDIX (Continued)}

3.19 e) You pretend you have not seen him. He probably does not want to deal with students outside of his workplace.

2.93 f) You ask if you can sit down with him and his kids and talk about different things.

$5.12 \mathrm{~g})$ You greet him and ask for an appointment with him the following day.

2.44 h) You greet him and offer to buy him and his kids coffee or sodas.

\section{Sample Problem, Including Mean Ratings for Each Response Strategy}

Across Experienced Participants in Study 2

You are in a very challenging class that emphasizes class discussion as the primary basis for a grade. You consider yourself intelligent, curious about ideas, but very shy, self-conscious, and nonassertive. You learn from the discussion around you, but it is very painful to feel that you have to contribute in order to get a good grade. You don't know anyone else in the class, and you don't know the professor. You have to get a good grade in this class to keep a decent GPA. Rate the quality of each of the following options:

\begin{tabular}{|c|c|c|c|c|c|c|}
\hline 1 & 2 & 3 & 4 & 5 & 6 & 7 \\
\hline Extremely & Very & Somewhat & Neither Bad & Somewhat & --- +---- & $\begin{array}{l}----+---- \\
\text { Extremely }\end{array}$ \\
\hline $\mathrm{Bad}$ & $\mathrm{Bad}$ & $\mathrm{Bad}$ & Nor Good & Good & Good & Good \\
\hline
\end{tabular}

3.39 a) Drop the class, and take another one that better fits your personality and one that you can succeed in.

5.63 b) Force yourself to ask at least one question in class each day, even if you think your question might make you look stupid.

2.64 d) Keep quiet. You can make up for what you lack in the discussion category by scoring well on quizzes and tests.

Table A1

Study 1: Correlations Among Covariates and Problem-Solving Scores

\begin{tabular}{lcccccc}
\hline \multicolumn{1}{c}{ Subscale } & 1 & 2 & 3 & 4 & 5 & 6 \\
\hline 1. Fluid intelligence & - & .14 & -.03 & -.09 & $-.17^{*}$ & $-.22^{* *}$ \\
2. Crystallized intelligence & & - & .05 & -.06 & -.06 & .02 \\
3. Analytical cognitive style & & & - & $-.36^{* * *}$ & .07 & $.17^{*}$ \\
4. Intuitive cognitive style & & & & - & .02 & .10 \\
5. Baseline problem-solving score & & & & - & $.36^{* * *}$ \\
6. Score on CSTKI problems & & & - \\
\hline Note-Includes "users" and control participants only. Higher problem-solving scores reflect \\
worse performance. ${ }^{*} p<.05 .{ }^{* *} p<.01$. & ${ }^{* * *} p<.001$.
\end{tabular}

Table A2

Study 2: Correlations Among Covariates and Problem-Solving Scores

\begin{tabular}{|c|c|c|c|c|}
\hline Subscale & 1 & 2 & 3 & 4 \\
\hline 1. Analytical cognitive style & - & -.09 & .15 & $.22^{*}$ \\
\hline 2. Intuitive cognitive style & & - & -.18 & $-.20^{*}$ \\
\hline 3. Baseline problem-solving score & & & - & $.49^{* * *}$ \\
\hline 4. Score on CSTKI problems & & & & - \\
\hline
\end{tabular}

(Manuscript received June 12, 2007;

revision accepted for publication September 21, 2007.) 